\title{
Growth-differentiation factor-15 and functional outcome after acute ischemic stroke
}

\author{
Klaus Gröschel · Sonja Schnaudigel · Frank Edelmann • Cord-Friedrich Niehaus • \\ Mark Weber-Krüger • Beatrice Haase $\cdot$ Rosine Lahno $\cdot$ Joachim Seegers $\cdot$ Katrin Wasser • \\ Janin Wohlfahrt · Dirk Vollmann · Raoul Stahrenberg $\cdot$ Rolf Wachter
}

Received: 3 November 2011/Revised: 6 December 2011/Accepted: 13 December 2011/Published online: 10 January 2012

(C) The Author(s) 2012. This article is published with open access at Springerlink.com

\begin{abstract}
Blood biomarkers may improve the performance in predicting early stroke outcome beyond well-established clinical factors. We investigated the value of growth-differentiation factor-15 (GDF-15) to predict functional outcome after 90 days in a prospectively collected patient cohort with symptoms of acute ischemic stroke. Two hundred eighty-one patients with symptoms of acute ischemic stroke were prospectively investigated. Serial blood samples for GDF-15 analysis were obtained after the admission of the patient, after 6 and $24 \mathrm{~h}$. Primary outcome was the dichotomized modified ranking scale (MRS) 90 days after the initial clinical event. Within the final study population (264 patients, mean age $70.3 \pm 12.7$ years, $55.3 \%$ male), National Institutes of Health Stroke Scale (NIH-SS) [odds ratio (OR) $1.269,95 \%$ confidence interval (CI) 1.141-1.412, $p<0.001$ ] and initial GDF-15 levels (OR 1.029, 95\% CI $1.007-1.053, p=0.011)$ were independently associated with a MRS $\geq 2$ after day 90 after multiple regression analysis. Growth-differentiation factor-15 levels increase with higher NIH-SS-tertiles $(p=0.005)$. Receiver-operator
\end{abstract}

K. Gröschel and S. Schnaudigel as well as R. Stahrenberg and R. Wachter contributed equally to this work.

K. Gröschel ( $\square)$

Department of Neurology, University of Mainz,

Langenbeckstr.1, 55131 Mainz, Germany

e-mail: klaus.groeschel@unimedizin-mainz.de

K. Gröschel · S. Schnaudigel $\cdot$ K. Wasser $\cdot$ J. Wohlfahrt Department of Neurology, University of Göttingen,

Göttingen, Germany

F. Edelmann - C.-F. Niehaus - M. Weber-Krüger - B. Haase ·

R. Lahno - J. Seegers · D. Vollmann - R. Stahrenberg ·

R. Wachter

Department of Cardiology and Pneumology,

University of Göttingen, Göttingen, Germany characteristic curves demonstrated a discriminatory accuracy to predict unfavourable stroke outcome of $0.629(95 \%$ CI 0.558-0.699), 0.753 (95\% CI 0.693-812) and 0.774 (95\% CI 0.717-0.832) for GDF-15, NIH-SS and the combination of these variables. The additional use of GDF-15 to NIH-SS ameliorates the model with a net reclassification index of $0.044(p=0.541)$ and integrated discrimination improvement of 0.034 ( $p=0.443)$. Growth-differentiation factor-15 as an acute stroke biomarker independently predicts unfavourable functional 90 day stroke outcome. Discriminatory value in addition to NIH-SS is only modestly distinct.

Keywords GDF-15 - Clinical trial · Ischemic stroke . TIA $\cdot$ Biomarker $\cdot$ Outcome

\section{Introduction}

Biomarkers in acute stroke may be an attractive tool to further improve the accuracy of predicting the individual patient's functional outcome. Against the background of the socioeconomic and clinical impact of stroke, an exact prediction of outcome is essential for patients, clinicians and researchers. Despite the strengths of clinical features such as well-established risk factors such as age, stroke severity or traditional cardiovascular risk factors, further refinement via biomarkers may be attractive because of the quick and easy measurement during routine stroke workup. Ideally, such a biomarker would add prognostic information that could otherwise not be obtained easily. Most of the studies that investigated the usefulness of biomarkers in acute stroke settings suffered from small sample sizes and disregarded important clinical features known to influence outcome [16].

Growth-differentiation factor-15 (GDF-15), a distant member of the transforming growth factor- $\beta$ cytokine super 
family, has increasingly been investigated as a prognostic biomarker in patients with cardiovascular diseases [10, 14]. Under normal conditions, GDF-15 is only weakly expressed in cardiovascular tissues, but its production increases sharply in different cardiovascular pathologies [8, 14, 17]. It has been shown to predict adverse cardiovascular events and total mortality beyond established risk factors in patients with cardiovascular diseases such as heart failure or coronary artery disease and in older community-dwelling adults free of known cardiovascular disease. [1, 3, 5] However, as cardiovascular burden may also contribute to functional outcome, biomarkers for cardiovascular disease may also give additional information on neurological functional outcome, as they have shown the most consistent associations with poor stroke outcome [16].

The aim of this study was to investigate the potential value of GDF-15 in predicting functional outcome after 90 days in a large prospective patient cohort with clinical symptoms of acute ischemic stroke.

\section{Methods}

\section{Study design}

In a single-center prospective trial (ISRCTN 46104198) [15], patients with symptoms of stroke starting less than $24 \mathrm{~h}$ ago were asked to give written consent for participation in the study. Participants were included after presenting to the Emergency Department of the University of Göttingen between March 2009 and February 2010. All patients received routine stroke care (cerebral imaging, cardiovascular workup including transthoracal echocardiography and Holter electrocardiogram, ultrasound of the brain-supplying arteries and routine blood analysis, including e.g. the lipid profile, C-reactive protein and creatinine levels) on a certified stroke unit.

\section{Blood sampling}

Nine $\mathrm{ml}$ of blood was drawn for the subsequent analysis of GDF-15 after at least 15 min of rest in the prone position from an antecubital or forearm vein. Samples were immediately centrifuged, and plasma was stored at $-80^{\circ} \mathrm{C}$ in aliquots labelled with patient ID and time of sampling for later analyses. Samples were drawn as soon as possible on presentation $(0 \mathrm{~h})$, and after 6 and $24 \mathrm{~h}$ to allow for the assessment of temporal trends early after the index event. After unfreezing of the samples, GDF-15 was measured with an electrochemiluminescence immunoassay on an automated Elecsys $(C)$ analyser (Roche Diagnostics GmbH, Mannheim, Germany). All measurements were done in a blinded fashion regarding diagnosis and outcome of the patients.
Clinical variables

Details about baseline patient characteristics have recently been reported [15]. In brief, data such as traditional cardiovascular risk factors were prospectively recorded in a predefined data sheet. Initial stroke severity and outcome after 3 months was assessed by video-trained physicians applying the National Institutes of Health Stroke Scale (NIH-SS) and modified Rankin Scale (MRS) respectively. Classification of stroke aetiology was done by experienced stroke neurologists using the widely accepted TOAST classification scheme.

The primary endpoint was the modified ranking scale assessed 90 days from baseline, defined as good functional outcome $(\mathrm{MRS} \leq 1)$ or ongoing disability (MRS $\geq 2$ ).

This study is in accordance with the Declaration of Helsinki and current ICH/GCP guidelines. Written informed consent was obtained from all participants or relatives. The study was approved by the Ethics Committee of the University of Göttingen.

Statistical analysis

Continuous values are expressed as mean $\pm \mathrm{SD}$, and nominal variables as count and percentages. Median values with the corresponding interquartile range (IQR) were computed for non-normally distributed variables. A twosided $t$ test was used for comparison of normally distributed variables and the non-parametric Kruskal-Wallis test for not normally distributed values. For comparisons of categorical data we used two-tailed chi-square statistics with Yates' correction or Fisher's exact test as applicable. Growth-differentiation factor-15 level comparison across different stroke severities (NIH-SS tertiles) was done using the Jonckheere-Terpstra test, which also tests for a monotonous trend across groups. Multiple binominal regression analyses were conducted for those variables with a $p<0.1$ on the univariate level to estimate the potential effect on the prediction of stroke outcome ( $p$ to enter $=0.05, p$ to leave $=0.1$ ) using additive and multiple interaction terms. Receiver-operator characteristics (ROC) curves were drawn and areas under the curves calculated for estimation of incremental prognostic information on functional outcome of the variables, which remained significant after multiple regression analysis. Comparisons of the area under the curves were done using the MedCalc for Windows (version 11.6.1.0, MedCalc Software, Mariakerke, Belgium). Furthermore, the net reclassification index and integrated discrimination improvement of the model were calculated according to the method of Pencina et al. [6, 11]. A $p$-value of less than 0.05 was considered to indicate a statistically significant difference. All statistical analyses were performed using 
SPSS (Version 19, SPSS Inc., Chicago, IL) unless otherwise stated.

\section{Results}

Two hundred eighty-one patients consented to participate in the study; one patient withdrew consent. For the present analysis, an additional seven had to be excluded because the diagnosis of suspected ischemic stroke was not confirmed during hospitalisation. One patient was lost to follow-up at day 90, and insufficient data on early blood biomarker sampling led to the exclusion of eight more patients. The characteristics of the 264 patients (mean age $70.3 \pm 12.7$ years, $55.3 \%$ male) included in the final analysis and stratified by 90-day outcome according to the MRS are presented in Table 1.

After univariate analysis patients with an unfavourable stroke outcome were statistically significantly older $(68.1 \pm 12.9$ vs. $73.2 \pm 11.8$ years, $p<0.001)$, presented with a higher NIH-SS (2 IQR 1-4 vs. 5 IQR 3-9, $p<0.001)$ and systolic blood pressure (BP) $(140.9 \pm 20.9$ vs. $147.0 \pm 23.8, p=0.028)$, and more frequently suffered from diabetes mellitus (17.9 vs. $33.6 \%, p=0.003)$ and coronary heart disease (11.9 vs. $22.1 \%, p=0.026)$. There was a difference according to the aetiology (classified by the TOAST classification scheme) of the stroke $(p<0.001)$, with a significantly higher proportion of suspected large artery aetiologies in the group with an unfavourable stroke outcome, whereas there were more strokes of unknown cause in the group with $\mathrm{MRS}<2$ after 90 days.

The time interval from the occurrence of clinical stroke symptoms to blood sampling did not differ between the two groups $(8.48 \pm 12.64$ vs. $6.63 \pm 6.42 \mathrm{~h}, \quad p=0.175)$. GDF-15 levels were significantly elevated within the group with an MRS $\geq 2$ after 90 days (1,056 IQR 815-1,481 vs. 1,372 IQR 912-2,381 ng/l, $p<0.001$ ), whereas there was no difference among acute inflammatory enzymes such as white blood cell count or C-reactive protein levels $(8.05 \pm$ $2.54 \times 10^{9}$ vs. $8.50 \pm 2.52 \times 10^{9} / 1, p=0.154$ and $7.49 \pm$ 25.48 vs. $12.57 \pm 28.70 \mathrm{mg} / \mathrm{l}, p=0.135$, respectively).
Table 1 Patient characteristics $(n=264)$

Patient characteristics dichotomized according to functional outcome after 90 days. Data are given as counts with percentages, mean with standard deviation or median with interquartile range depending on appropriateness $N I H$-SS National Institutes of Health Stroke Scale, $r t-P A$ recombinant tissue plasminogen activator, $B P$ blood pressure, $G D F-15$ growth-differentiation factor-15, $L D L-C$ low-density lipoprotein cholesterol, $H D L-C$ high-density lipoprotein cholesterol

* Included into multivariate regression analysis $(p<0.1)$

$\uparrow$ Remained significant after multiple regression analysis

\begin{tabular}{|c|c|c|c|}
\hline & $\mathrm{MRS} \leq 1$ & $\mathrm{MRS} \geq 2$ & $P$-value \\
\hline Demographic data & $n=151$ & $n=113$ & \\
\hline Age (years) & $68.1( \pm 12.9)$ & $73.2( \pm 11.8)$ & $0.001^{*}$ \\
\hline Female gender & $66(43.7 \%)$ & $52(46.0 \%)$ & 0.139 \\
\hline NIH-SS on admission & $2(1-4)$ & $5(3-9)$ & $<0.001^{* \dagger}$ \\
\hline rt-PA lysis & $7(4.6 \%)$ & $12(10.6 \%)$ & $0.065^{*}$ \\
\hline BP systolic (mmHG) & $140.9( \pm 20.9)$ & $147.0( \pm 23.8)$ & $0.028 *$ \\
\hline BP diastolic (mmHG) & $79.5( \pm 12.6)$ & $80.7( \pm 15.0)$ & 0.172 \\
\hline Heart rate (beats/minute) & $72.3( \pm 13.9)$ & $74.6( \pm 15.7)$ & 0.211 \\
\hline Temperature $\left({ }^{\circ} \mathrm{C}\right)$ & $36.8( \pm 0.4)$ & $36.8( \pm 0.3)$ & 0.663 \\
\hline \multicolumn{3}{|c|}{ TOAST classification scheme for stroke aetiology } & $0.001 *$ \\
\hline Large artery & $20(13.2 \%)$ & $28(24.8 \%)$ & \\
\hline Cardio embolic & $37(24.5 \%)$ & $40(35.4 \%)$ & \\
\hline Small vessel & $18(11.9 \%)$ & $12(10.6 \%)$ & \\
\hline Unknown & $74(49.0 \%)$ & $29(25.7 \%)$ & \\
\hline Rare/other causes & $2(1.3 \%)$ & $4(3.5 \%)$ & \\
\hline Arterial hypertension & $108(71.5 \%)$ & $89(78.8 \%)$ & 0.181 \\
\hline Diabetes mellitus & $27(17.9 \%)$ & $38(33.6 \%)$ & $0.003^{*}$ \\
\hline Smoking, current & $34(22.5 \%)$ & $22(19.5 \%)$ & 0.165 \\
\hline Hyperlipidemia & $49(32.5 \%)$ & $41(36.3 \%)$ & 0.516 \\
\hline Coronary heart disease & $18(11.9 \%)$ & $25(22.1 \%)$ & $0.026^{*}$ \\
\hline Peripheral artery disease & $4(2.6 \%)$ & $3(2.7 \%)$ & 0.998 \\
\hline GDF-15 (ng/l) & $1056(815-1,481)$ & $1372(912-2,381)$ & $<0.001 * \dagger$ \\
\hline White blood cell count $\left(\times 10^{9} / 1\right)$ & $8.05( \pm 2.54)$ & $8.50( \pm 2.52)$ & 0.154 \\
\hline C-reactive protein (mg/l) & $7.49( \pm 25.48)$ & $12.57( \pm 28.70)$ & 0.135 \\
\hline Cholesterol (mmol/l) & $5.06( \pm 1.05)$ & $4.91( \pm 1.09)$ & 0.277 \\
\hline LDL-C (mmol/l) & $3.30( \pm 0.86)$ & $3.25( \pm 0.90)$ & 0.636 \\
\hline HDL-C (mmol/l) & $1.36( \pm 0.36$ & $1.26( \pm 0.47)$ & $0.062 *$ \\
\hline Triglyceride (mmol/l) & $1.41( \pm 0.75)$ & $1.50( \pm 0.66)$ & 0.363 \\
\hline
\end{tabular}


There was a significant increase in GDF-15 levels with rising stroke severity (Jonckheere-Terpstra test $p<0.005$ ). Growth-differentiation factor-15 levels decreased from the first blood sampling after presentation of the patients to the emergency department to the sampling after 6 and $24 \mathrm{~h}$ $(1,672 \pm 1,709,1,541 \pm 1,293$ and $1,458 \pm 1,123 ; p$ for trend $<0.001)$.

After applying binominal multivariate analysis with variables that were imbalanced $(p<0.1)$ on the univariate level (age, NIH-SS, rt-PA lysis, $\mathrm{BP}_{\text {sys }}$, TOAST classification, diabetes mellitus, coronary heart disease, GDF-15, HDL cholesterol), the variables NIH-SS [odds ratio (OR) 1.269 , 95\% confidence interval (CI) 1.141-1.412, $p<0.001]$ and GDF-15 (OR per $100 \mathrm{ng} / 1$ 1.029, 95\% CI $1.007-1.053, p=0.011)$ remained significant for predicting an unfavourable outcome after 3 months (Table 2).

Table 2 Results of the multivariate analysis

\begin{tabular}{lllr}
\hline & Odds ratio & $\begin{array}{l}\text { 95\% Confidence } \\
\text { interval }\end{array}$ & $P$-value \\
\hline NIH-SS $_{\text {on admission }}$ & 1.269 & $1.141-1.412$ & $<0.001$ \\
GDF-15 per 100 ng/1 & 1.029 & $1.007-1.053$ & 0.011 \\
\hline
\end{tabular}

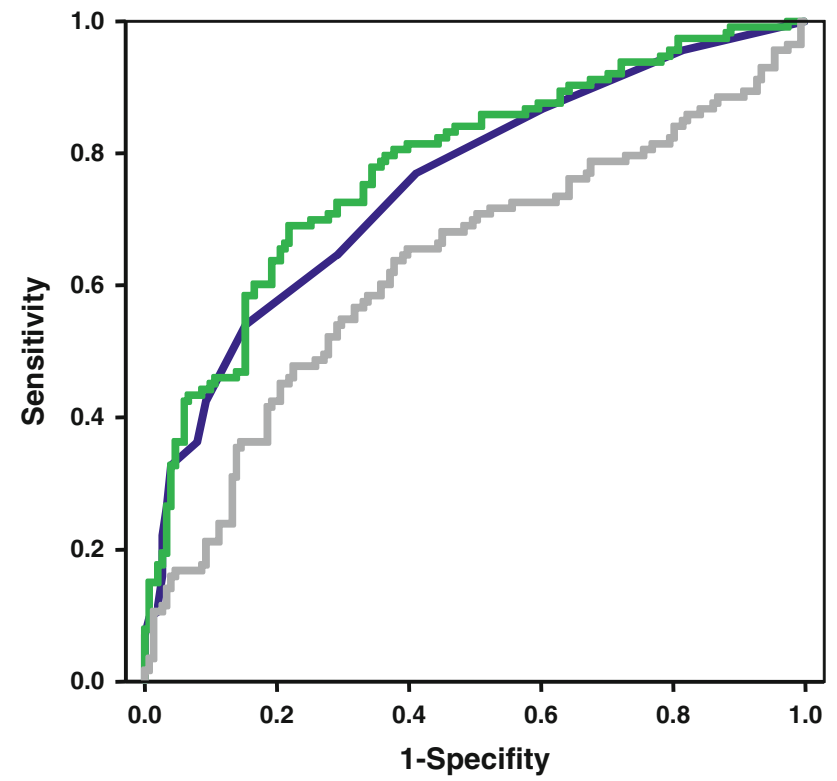

Fig. 1 Receiver-operating characteristics curve (ROC) for NIH-SS, GDF-15 and the combination of the latter for the prediction of 90-day stroke outcome (MRS $>1$ ). Area under the curve improved from $0.629(95 \%$ CI $0.558-0.699 ; p<0.001)$ and $0.753(95 \%$ CI $0.693-812 ; p<0.001$ ) for GDF-15 (grey) and NIH-SS (blue) to 0.774 (95\% CI $0.717-0.832 ; p<0.001$ ) for the combination of the two variables (green). The combination of the two variables was better than GDF-15 ( $p=0.002)$ alone, but not statistically significantly compared to the NIH-SS on its own $(p=0.621)$
Receiver-operating characteristics curve (ROC) for GDF-15, NIH-SS and the combination of the latter for the prediction of a 90-day stroke outcome of MRS $>1$ resulted in a ROC of 0.629 (95\% CI 0.558-0.699; $p<0.001), 0.753$ $(95 \%$ CI $0.693-812 ; p<0.001)$ and $0.774(95 \%$ CI $0.717-0.832 ; p<0.001$ ), respectively (Fig. 1 ). The combination of the two variables yielded a better discriminative power than GDF-15 $(p=0.002)$ alone, but not more discriminative power than NIH-SS $(p=0.621)$ on its own.

The net reclassification index of the model with the two variables GDF-15 and NIH-SS improved by 0.044 in comparison to the prediction with NIH-SS alone $(p=0.541)$; the integrated discrimination improvement of the model with the additional use of GDF-15 was $0.034(p=0.443)$.

\section{Discussion}

The aim of our study was to evaluate the value of the novel cardiovascular biomarker GDF-15 for the prediction of functional outcome after 90 days in patients with symptoms of acute cerebral ischaemia. We could demonstrate that GDF-15 levels rise with increasing stroke severity, decreases within the 1 st day after the initial symptoms and that GDF-15 is capable of predicting unfavourable stroke outcome. In addition to well-established clinical variables such as the baseline NIH-SS, the additional predictive power of GDF-15 was only modest and non-significant.

Many efforts have been made to investigate different biomarkers to predict functional outcome after stroke, highly relevant information for clinicians, researchers and patients to optimise care and allocation of healthcare resources [7]. However, most of the studies were small and did not adjust for relevant and basic clinical information such as age or stroke severity. Where calculated, their incremental predictive power over e.g. the NIH-SS was only modest or absent [16]. Growth-differentiation factor- 15 has been identified as a novel biomarker holding prognostic information in different cardiovascular diseases [5, 8-10, 17]. Moreover, it has recently been shown to be elevated after experimental brain injury [13] and in a middle cerebral artery occlusion rat model of ischemic stroke [12]. An upregulation of GDF-15 was observed and neurons could be identified as the predominant source of expression, although the exact relevance of this upregulation still remains unclear.

Within our study, we were able to support the assumption that GDF-15 has a potential to predict outcome after stroke. We could demonstrate that GDF-15 levels after acute cerebral ischaemia are elevated, positively correlated with the severity of stroke symptoms and decrease over time, implicating a possible association with the extent of brain tissue damage. Our data confirm a recently published case series of 57 stroke patients, where elevated GDF-15 
levels were associated with an adverse outcome after stroke [18]. Now we were able to prove this in a larger patient cohort that was less selected (e.g. patients with thrombolysis were excluded from participation in the former study) and thus translate these provisional findings into a realworld scenario.

The exact role of GDF-15 after stroke is currently being discussed and may rely on different pleiotropic modes of action. Growth-differentiation factor-15 has been hypothesised to be a downstream marker that integrates several different pathways of cardiovascular stress [8]. Apart from its direct association with brain damage [12, 13], it may therefore be integratively indicative of acute cardiovascular pertubations secondary to the cerebral ischemic event. In this manner, GDF-15 may transport information on stroke severity as well as the cardiovascular consequences that are only partially captured by clinical scores that describe stroke severity. However, it should be kept in mind that GDF-15 is also elevated in other, non-cardiovascular entities [2, 4]. It might therefore be to some extent an unspecific marker for additional non-cardiovascular comorbidities that portend functional impairment.

Despite the strengths of our study we are well aware of some limitations: although data collection was prospective and blinded to the determination of the blood analysis, the current analysis was done in a retrospective fashion and may be subjected to bias. A prospective validation in a larger cohort is therefore recommended.

Our data corroborate that GDF-15 is a potential biomarker in patients with cerebral ischaemia that indicates functional 90-day outcome like the baseline stroke severity expressed by the NIH-SS. We suggest further investigations of GDF-15 in larger cohorts to develop recommendations on how to instrumentalize this promising marker in clinical practice and tailor therapy according to an optimised early risk stratification.

Acknowledgments Source of funding: This work was supported by grants from the German Federal Ministry of Education and Research (German Heart Failure Network, TP 7 (FKZ 01GI0205) and clinical trial program Aldo-DHF (FKZ 01KG0506)). This study was supported by an unrestricted grant from Roche Diagnostics, Mannheim, Germany.

\section{Conflicts of interest None.}

Open Access This article is distributed under the terms of the Creative Commons Attribution Noncommercial License which permits any noncommercial use, distribution, and reproduction in any medium, provided the original author(s) and source are credited.

\section{References}

1. Anand IS, Kempf T, Rector TS, Tapken H, Allhoff T, Jantzen F, Kuskowski M, Cohn JN, Drexler H, Wollert KC (2010) Serial measurement of growth-differentiation factor-15 in heart failure: relation to disease severity and prognosis in the Valsartan heart failure trial. Circulation 122:1387-1395

2. Bauskin AR, Brown DA, Kuffner T, Johnen H, Luo XW, Hunter M, Breit SN (2006) Role of macrophage inhibitory cytokine-1 in tumorigenesis and diagnosis of cancer. Cancer Res 66:4983-4986

3. Bonaca MP, Morrow DA, Braunwald E, Cannon CP, Jiang S, Breher S, Sabatine MS, Kempf T, Wallentin L, Wollert KC (2011) Growth differentiation factor-15 and risk of recurrent events in patients stabilized after acute coronary syndrome: observations from PROVE IT-TIMI 22. Arterioscler Thromb Vasc Biol 31:203-210

4. Brown DA, Moore J, Johnen H, Smeets TJ, Bauskin AR, Kuffner T, Weedon H, Milliken ST, Tak PP, Smith MD, Breit SN (2007) Serum macrophage inhibitory cytokine 1 in rheumatoid arthritis: a potential marker of erosive joint destruction. Arthr Rheum 56:753-764

5. Daniels LB, Clopton P, Laughlin GA, Maisel AS, Barrett-Connor E (2011) Growth-differentiation factor-15 is a robust, independent predictor of 11-year mortality risk in community-dwelling older adults: the rancho bernardo study. Circulation 123:21012110

6. Hlatky MA, Greenland P, Arnett DK, Ballantyne CM, Criqui MH, Elkind MS, Go AS, Harrell FE Jr, Hong Y, Howard BV, Howard VJ, Hsue PY, Kramer CM, McConnell JP, Normand SL, O'Donnell CJ, Smith SC Jr, Wilson PW (2009) Criteria for evaluation of novel markers of cardiovascular risk: a scientific statement from the American Heart Association. Circulation 119:2408-2416

7. Katan M, Fluri F, Morgenthaler NG, Schuetz P, Zweifel C, Bingisser R, Muller K, Meckel S, Gass A, Kappos L, Steck AJ, Engelter ST, Muller B, Christ-Crain M (2009) Copeptin: a novel, independent prognostic marker in patients with ischemic stroke. Ann Neurol 66:799-808

8. Kempf T, von Haehling S, Peter T, Allhoff T, Cicoira M, Doehner W, Ponikowski P, Filippatos GS, Rozentryt P, Drexler H, Anker SD, Wollert KC (2007) Prognostic utility of growth differentiation factor-15 in patients with chronic heart failure. J Am Coll Cardiol 50:1054-1060

9. Lankeit M, Kempf T, Dellas C, Cuny M, Tapken H, Peter T, Olschewski M, Konstantinides S, Wollert KC (2008) Growth differentiation factor-15 for prognostic assessment of patients with acute pulmonary embolism. Am J Respir Crit Care Med 177:1018-1025

10. Lind L, Wallentin L, Kempf T, Tapken H, Quint A, Lindahl B, Olofsson S, Venge P, Larsson A, Hulthe J, Elmgren A, Wollert $\mathrm{KC}$ (2009) Growth-differentiation factor-15 is an independent marker of cardiovascular dysfunction and disease in the elderly: results from the prospective investigation of the vasculature in uppsala seniors (PIVUS) study. Eur Heart J 30:2346-2353

11. Pencina MJ, D’Agostino RB Sr, D’Agostino RB Jr, Vasan RS (2008) Evaluating the added predictive ability of a new marker: from area under the ROC curve to reclassification and beyond. Stat Med 27:157-172

12. Schindowski K, Bohlenund HO, Strelau J, Ridder DA, Herrmann O, Schober A, Schwaninger M, Unsicker K (2011) Regulation of GDF-15, a distant TGF-beta superfamily member, in a mouse model of cerebral ischemia. Cell Tissue Res 343:399-409

13. Schober A, Bottner M, Strelau J, Kinscherf R, Bonaterra GA, Barth M, Schilling L, Fairlie WD, Breit SN, Unsicker K (2001) Expression of growth differentiation factor-15/macrophage inhibitory cytokine-1 (GDF-15/MIC-1) in the perinatal, adult, and injured rat brain. J Comp Neurol 439:32-45

14. Stahrenberg R, Edelmann F, Mende M, Kockskamper A, Dungen HD, Luers C, Binder L, Herrmann-Lingen C, Gelbrich G, Hasenfuss G, Pieske B, Wachter R (2010) The novel biomarker 
growth differentiation factor 15 in heart failure with normal ejection fraction. Eur J Heart Fail 12:1309-1316

15. Stahrenberg R, Weber-Krüger M, Seegers J, Edelmann F, Lahno R, Haase B, Mende M, Wohlfahrt J, Kermer P, Vollmann D, Hasenfuss G, Gröschel K, Wachter R (2010) Enhanced detection of paroxysmal atrial fibrillation by early and prolonged continuous holter monitoring in patients with cerebral ischemia presenting in sinus rhythm. Stroke 41:2884-2888

16. Whiteley W, Chong WL, Sengupta A, Sandercock P (2009) Blood markers for the prognosis of ischemic stroke: a systematic review. Stroke 40:e380-e389
17. Wollert KC, Kempf T, Peter T, Olofsson S, James S, Johnston N, Lindahl B, Horn-Wichmann R, Brabant G, Simoons ML, Armstrong PW, Califf RM, Drexler H, Wallentin L (2007) Prognostic value of growth-differentiation factor-15 in patients with non-STelevation acute coronary syndrome. Circulation 115:962-971

18. Worthmann H, Kempf T, Widera C, Tryc AB, Goldbecker A, Ma YT, Deb M, Tountopoulou A, Lambrecht J, Heeren M, Lichtinghagen R, Wollert KC, Weissenborn K (2011) Growth differentiation factor 15 plasma levels and outcome after ischemic stroke. Cerebrovasc Dis 32:72-78 